\title{
Ein Index zur Trennung von individuellem und strukturellem Wandel zwischen zwei Meßzeitpunkten einer Panelbefragung
}

\section{Thomas Klein}

Institut für Medizinische Informatik und Systemforschung (MEDIS) der Gesellschaft für Strahlen- und Umweltforschung mbH (GSF), Ingolstädter Landstraße 1, D-8042 Neuherberg

Zusammenfassung: In den zurückliegenden Jahren sind in der empirischen Sozialforschung in zunehmendem Maße Panelerhebungen durchgeführt worden. Dabei stellt sich häufig die Frage, ob Veränderungen von einer Befragung zur nächsten individueller Natur sind oder als sozialstruktureller Wandel interpretiert werden können. Der vorliegende Beitrag diskutiert zwei bislang in der Literatur vorgeschlagene Maße zur Messung des Anteils an zufälliger Veränderung hinsichtlich ihrer Validität und entwickelt aus ihrer Kritik eine verbesserte Maßzahl. Dieser verbesserte Index ist in dreifacher Hinsicht nützlich: Er zeigt an, inwieweit sozialstruktureller Wandel als „stabil“ bezeichnet werden kann oder von gegenläufigen Tendenzen durchsetzt ist. Er kann darüber hinaus zur Beurteilung der Wirksamkeit sozialpolitischer Maßnahmen beitragen. Schließlich gibt er u. U. einen Aufschluß über die (Retest-) Reliabilität der verwendeten Fragen, wenn man vermuten kann, daß real gar keine Veränderung stattgefunden hat.

\section{Einleitung}

In den zurückliegenden Jahren sind in der empirischen Sozialforschung in zunehmendem Maße Zeitverlaufserhebungen durchgeführt worden, die sich nicht mehr - wie Querschnittserhebungen auf die Erfassung eines einzelnen Zeitpunkts geschränken, sondern darüber hinaus auf die Veränderungen im Zeitablauf, die Ereignisse und die Zustandsdauer, abzielen. Wegen der Probleme, die mit retrospektiven Erhebungen verbunden sind, der aus verschiedenen Gründen unzuverlässigeren Angaben und der geringeren Bereinigungsmöglichkeiten (s. z. B. Tölke 1980), werden Zeitverläufe häufig auf der Basis von Wiederholungsbefragungen erfaßt. Dabei stellt sich die Frage, ob Veränderungen von einer Befragung zur nächsten individueller Natur sind oder ob sie einen (sozial-) strukturellen Wandel anzeigen. Im Rahmen einer explorativen Datenanalyse informiert die Aufschlüsselung von individueller versus struktureller Veränderung bei verschiedenen Variablen gleichzeitig über die Bereiche, in denen struktureller Wandel festgestellt werden kann und an dem eine Analyse des Wandels daher ansetzen sollte. Schließlich kann die Aufspaltung der Gesamtveränderung in eine individuelle und eine strukturelle Komponente als Indiz für die (Retest-)Reliabilität der verwendeten Fragen gewertet werden, wenn in dem abgedeckten Zeitraum (weil er zu kurz ist oder aus sonstigen Gründen) keine reale Veränderung anzunehmen ist.

Zur Beantwortung der Frage nach dem Anteil individueller versus struktureller Veränderung sind von Kessler und Greenberg (1981) und von
Kirschner (1986) zwei varianzanalytische Maße für den Anteil individueller und den verbleibenden Anteil struktureller Veränderung entwickelt worden. Der vorliegende Beitrag diskutiert beide MaBe (Punkt 2) und entwickelt aus der Kritik eine verbesserte Maßzahl für die Komponente individueller bzw. struktureller Veränderung (Punkt 3).

\section{Eine Diskussion der Kessler-Greenberg- Zerlegung der Varianz der Meßdifferenz zwischen zwei Meßzeitpunkten und des normierten Maßes für individuellen Wandel von Kirschner}

\subsection{Beschreibung}

Das von Kessler und Greenberg (1981: 51) vorgeschlagene Maß beruht auf der Meßdifferenz zwischen zwei Zeitpunkten einer Panelbefragung. Die Varianz der Meßdifferenz läßt sich in zwei Summanden zerlegen, von denen einer als Maß für strukturellen Wandel und der andere als $\mathrm{Maß}$ für individuelle Veränderung interpretiert wird. Bezeichnet man die Meßwerte zu den Zeitpunkten 1 und 2 mit $x_{1}$ bzw. $x_{2}$ und die Varianz der Meßdifferenz mit $s\left(x_{1}-x_{2}\right)^{2}$, so läßt sich die Kessler-Greenberg-Zerlegung gemäß

$$
\begin{aligned}
& \mathrm{s}\left(\mathrm{x}_{1}-\mathrm{x}_{2}\right)^{2}= \\
& \mathrm{s}\left(\mathrm{x}_{1}\right)^{2}\left(\mathrm{~b}\left(\mathrm{x}_{2}, \mathrm{x}_{2}\right)-1\right)^{2}+\mathrm{s}\left(\mathrm{x}_{2}\right)^{2}\left(1-\mathrm{r}\left(\mathrm{x}_{1}, \mathrm{x}_{2}\right)^{2}\right)
\end{aligned}
$$

wiedergeben, wobei mit $s\left(x_{1}\right)^{2}$ bzw. $s\left(x_{2}\right)^{2}$ die Varianzen in den Zeitpunkten 1 bzw. 2 dargestellt sind, mit $r\left(x_{1}, x_{2}\right)$ der Korrelationskoeffizient $z w i-$ schen beiden Meßzeitpunkten und mit $b\left(x_{2}, x_{1}\right)$ der 
Regressionskoeffizient mit der unabhängigen Variable $\mathrm{x}_{1}$. Hierbei soll der erste Summand die strukturelle Veränderung und der zweite die individuelle Veränderung anzeigen. Der Anteil individueller Veränderung an der Gesamtvarianz läßt sich daher entsprechend

(2) $\quad$ ind $=\frac{s\left(x_{2}\right)^{2}\left(1-r\left(x_{1}, x_{2}\right)^{2}\right)}{s\left(x_{1}-x_{2}\right)^{2}}$

ausdrücken. Tabelle 1 zeigt (jeweils in der obersten Zeile), wie der aus Gleichung (2) berechnete Anteil individueller Varianz von dem Verhältnis der Standardabweichungen $\mathbf{s}\left(\mathrm{x}_{2}\right) / \mathrm{s}\left(\mathrm{x}_{1}\right)$ und von dem Korrelationskoeffizienten abhängt.
Die dargestellte Kessler-Greenberg-Zerlegung weist, wie Kirschner (1986) darlegt, eine Reihe von Nachteilen auf, die einer Interpretation im Sinne des Anteils individueller Veränderung entgegenstehen. Am gewichtigsten ist dabei der Nachteil, daß eine Vergrößerung und eine Verkleinerung der Varianz zum zweiten Meßzeitpunkt von der Kessler-Greenberg-Zerlegung ungleich beurteilt werden (s. Kirschner 1986: 28). Wie aus Tabelle 1 ersichtlich, führt eine geringere Varianz im zweiten Meßzeitpunkt $\left(\mathrm{s}\left(\mathrm{x}_{2}\right) / \mathrm{s}\left(\mathrm{x}_{1}\right)<1\right)$ unabhängig von $r\left(x_{1}, x_{2}\right)$ zu einer Verringerung des Anteils individueller Varianz, während für eine größere Varianz zunächst eine Vergrößerung des individuellen Anteils ausgewiesen wird. Auch eine vergrö-

Tabelle 1 Der Kessler-Greenberg-Anteil individueller Varianz (ind), das normierte Maß für individuelle Veränderung (rel) und der Individualindex für die Komponente individueller Veränderung (IK) nach dem Korrelationskoeffizienten und dem Verhältnis der Standardabweichungen.

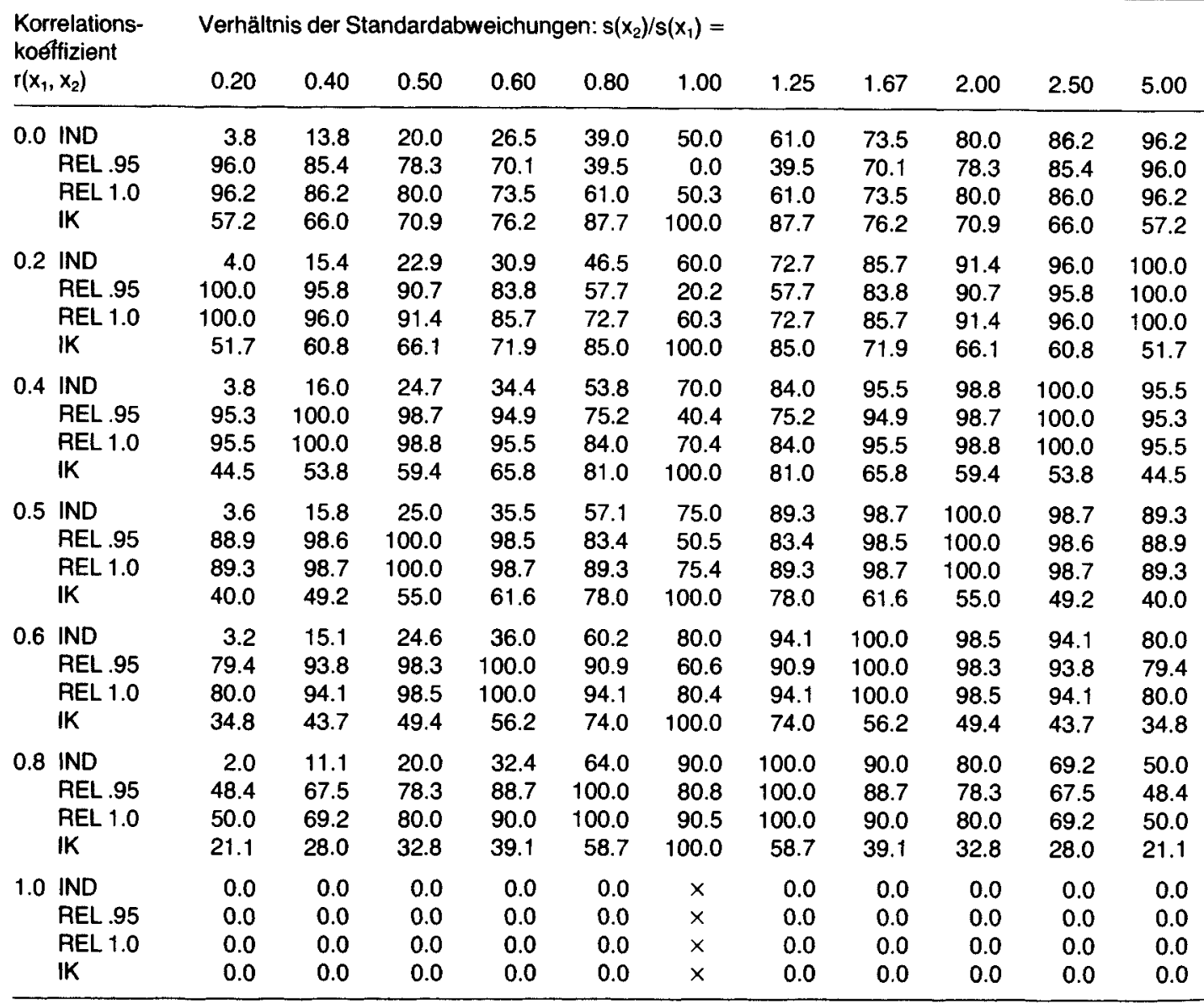


Berte Varianz kann jedoch bei konstantem $r\left(\mathrm{x}_{1}, \mathrm{x}_{2}\right)$ strukturell bedingt sein, so daß die Kessler-Greenberg-Zerlegung zu „krassen Fehlinterpretationen“ (Kirschner 1986: 28) führen kann.

Gleichzeitig weist Kirschner (1986: 27) darauf hin, daß der Anteil individueller Varianz in dem forschungspraktisch relevanten Bereich eines hohen Korrelationskoeffizienten und eines Varianzverhältnisses nahe bei 1 durchgehend sehr hoch erscheint und auch bei geringerer Korrelation kaum unter 50 Prozent absinken kann (s. Tabelle 1). Angesichts dieser und anderer Nachteile ${ }^{1}$ entwikkelt Kirschner (1986) auf der Grundlage der dargestellten Varianzzerlegung ein sogenanntes normiertes Maß für den Anteil individueller Veränderung, das den Kessler-Greenberg-Anteil individueller Veränderung bei konstantem Varianzverhältnis und variierendem Korrelationskoeffizienten zwischen 0 und 0,95 auf den möglichen Wertebereich bezieht. Bezeichnet man bei „durchlaufendem" Korrelationskoeffizienten $0 \leq \mathrm{r}\left(\mathrm{x}_{1}, \mathrm{x}_{2}\right) \leq$ 0,95 den minimalen Anteil individueller Veränderung mit indmin und den maximalen Anteil mit indmax, so ist das normierte bzw. relativierte $\mathrm{Ma} B$ von Kirschner gemäß

$$
\text { rel }=\frac{\text { ind }- \text { indmin }}{\text { indmax }- \text { indmin }}
$$

definiert. Die Abhängigkeit des normierten Maßes von $s\left(x_{2}\right) / s\left(x_{1}\right)$ und von $r\left(x_{1}, x_{2}\right)$ ist ebenfalls aus Tabelle 1 (jeweils zweite Zeile) zu entnehmen. Die Tabelle zeigt bei unterschiedlichen Korrelationskoeffizienten für rel einen größeren Wertebereich als für ind. Der mit rel gemessene Anteil individueller Veränderung verhält sich außerdem „symmetrisch" hinsichtlich des Verhältnisses der Standardabweichungen $\mathrm{zu}$ beiden Meßzeitpunkten, was heißt, daß für $s\left(x_{2}\right) / s\left(x_{1}\right)$ und $s\left(x_{1}\right) / s\left(x_{2}\right)$ bei gleichem $r\left(x_{1}, x_{2}\right)$ gleiche Anteile individueller Veränderung ausgewiesen werden.

\footnotetext{
${ }^{1}$ Ein weiterer Nachteil besteht darin, daß von vornherein „eingebaute“ strukturelle bzw. individuelle Veränderungen von der Kessler-Greenberg-Zerlegung nicht zutreffend bemerkt werden (s. Kirschner 1986: 24). Andere Kritikpunkte erscheinen hingegen kaum zutreffend. So ist es z. B. kein Nachteil, wenn unterschiedliche Wertekombinationen (Überströmmatritzen) zu gleichen Werten der Standardabweichungen, demselben Korrelationskoeffizienten und dabei demselben Anteil individueller Veränderung führen. Jeder arithmetische Durchschnitt kann sich aus unterschiedlichen Werten berechnen. Im übrigen weist in dem Kirschnerschen Kritikbeispiel auch sein normiertes Maß gleiche Werte auf.
}

\subsection{Eine Validitätsbeurteilung anhand von Extremfällen}

Für eine systematische (Validitäts-)Diskussion beider Maße sind zwei von Kirschner (1986: 23) angeregte Leitfragen sinnvoll, die Frage nämlich, ob

- wenigstens Extremfälle von Veränderung ,richtig" zugeordnet und ob

- markante Unterschiede von Veränderungen "richtig“ wiedergegeben werden.

In den Schaubildern 1 und 2 sind solche Extremfälle graphisch dargestellt. Der Anschaulichkeit halber sind ganz einfache Beispiele mit jeweils acht Wertepaaren ausgewählt worden. In allen Schaubildern gibt die Horizontalachse den Meßwert zum ersten und die Vertikalachse den zum zweiten Zeitpunkt wieder. Findet keinerlei Veränderung statt, sind alle Wertepaare auf der 45-Grad-Linie angesiedelt. Findet eine rein individuelle Veränderung statt, so sind die Wertepaare in einem Streifen (,Rauschen") um die Winkelhalbierende angeordnet. Bei einer teilweise strukturellen Veränderung hat man dagegen von einer Punktwolke auszugehen, die aus dem Streifen um die Winkelhalbierende ,herausdreht". Angesichts einer völlig strukturellen Veränderung würden dagegen alle Wertepaare auf einer Linie liegen, die steiler oder auch flacher als die Winkelhalbierende verläuft ${ }^{2}$.

Betrachten wir zunächst den Extremfall in Schaubild $1 \mathrm{a}$, in dem offensichtlich keinerlei Veränderung stattfindet und ein Anteil an Veränderung somit nicht definiert ist. Wie aus Tabelle $2 \mathrm{zu}$ entnehmen, geben sowohl ind als auch rel diesen Extremfall richtig wieder - beide Maße sind für $r\left(x_{1}, x_{2}\right)=1$ und $s\left(x_{2}\right) / s\left(x_{1}\right)=1$ nicht definiert. ${ }^{3}$ In Schaubild $1 \mathrm{~b}$ ist ein in gewisser Weise entgegengesetzter Extremfall dargestellt, in dem die auftretende Veränderung ohne jede Systematik als bloBes „Rauschen“ um die Winkelhalbierende in Erscheinung tritt. Eine „richtige“, d.h. valide Messung müßte daher die Veränderung zu 100 Prozent als individuelle Veränderung verbuchen. Weder

\footnotetext{
${ }^{2}$ Denkbar ist auch, daß rein additive Struktureffekte auftreten, die zu einer 45-Grad-Linie ober- oder unterhalb der Winkelhalbierenden führen. Dieser Fall wird von Kirschner nicht analysiert.
}

Kirschner (1986: 28ff) „verzichtet" auf eine Berechnung für Korrelationskoeffizienten größer als 0,95. Wie weiter unten diskutiert, ist jedoch dieser Verzicht und die Grenze dieses Verzichts von ganz elementarer Bedeutung für den Wert von rel. Auch ohne diesen Verzicht ist jedoch rel für ein Varianzverhältnis und einen Korrelationskoeffizienten von jeweils 1 nicht definiert. 
1a)

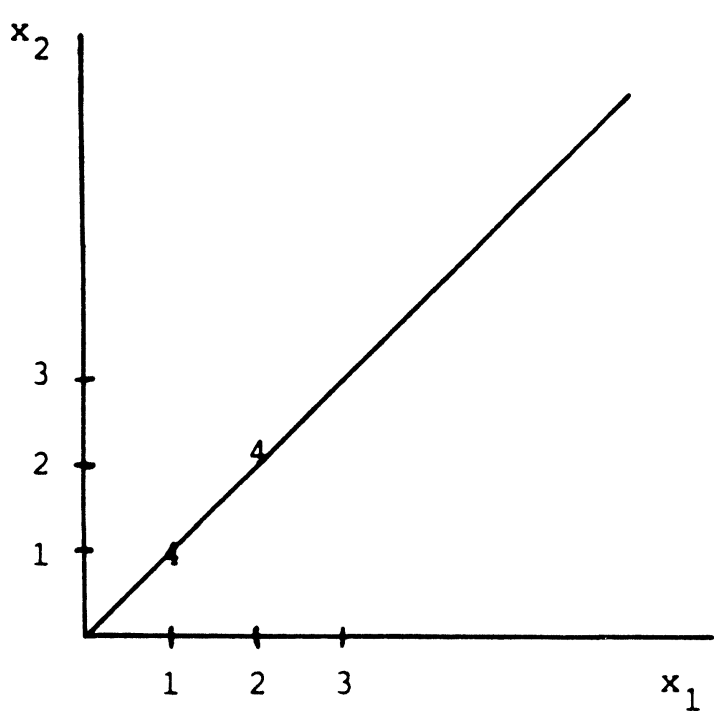

1c)

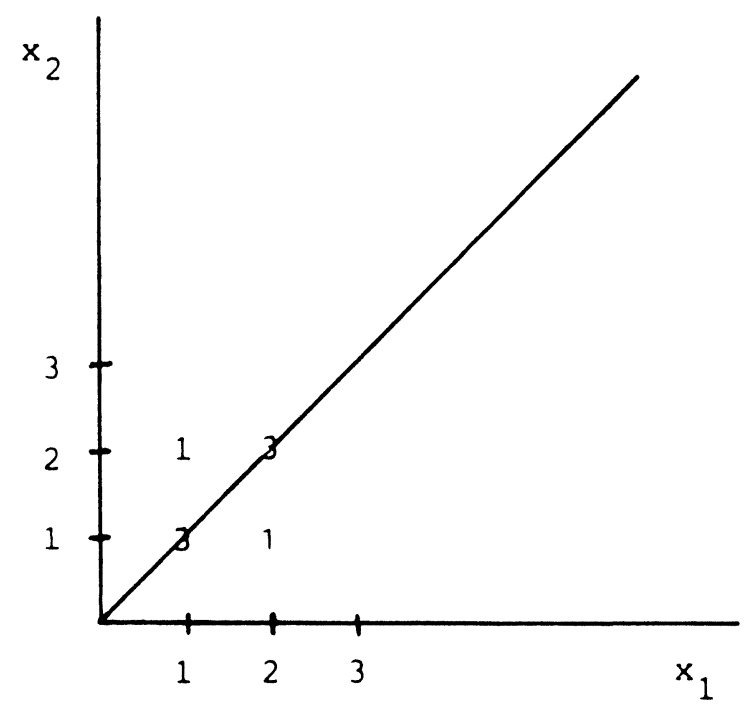

1b)

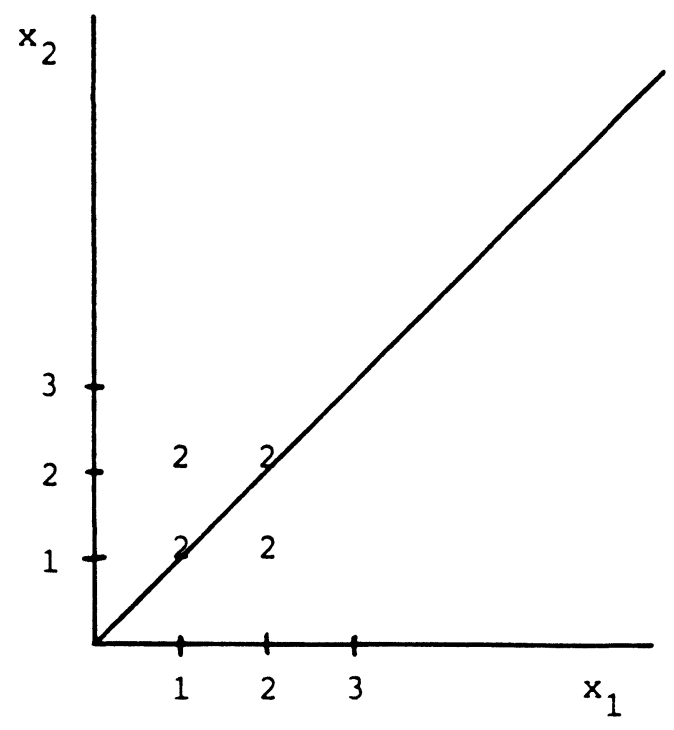

1d)

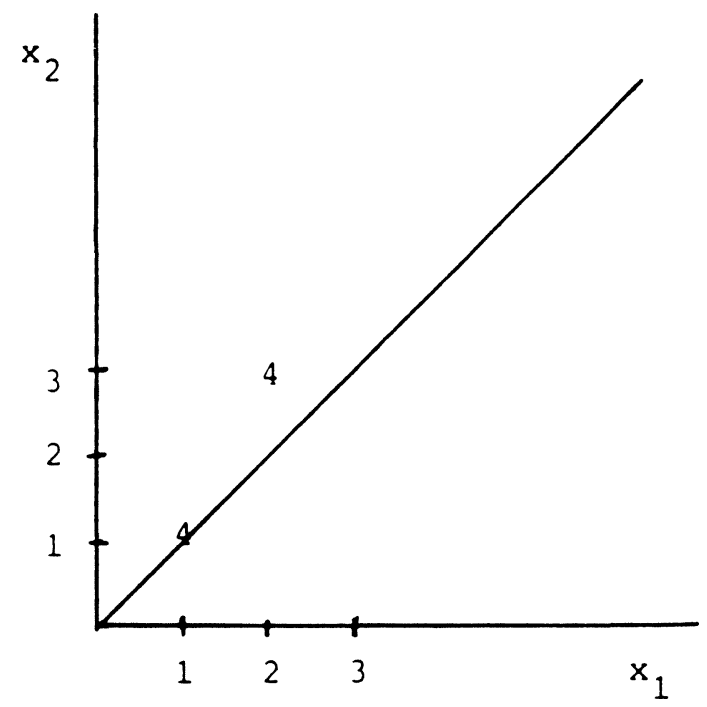

Schaubild 1 Extrembeispiele für Veränderungen zwischen zwei Meßzeitpunkten.

Jeweils sind 8 Wertepaare dargestellt. Die Zahlen geben die Häufigkeit der jeweiligen Wertekombination wieder, also: 1 = 1 Beobachtung, 2 = 2 Beobachtungen usw.

Quelle: eigene Zusammenstellung 
Tabelle 2 Extremfälle und markante Unterschiede des Anteils individueller Veränderung zwischen zwei Meßzeitpunkten einer Panelbefragung.

\begin{tabular}{lrllllll}
\hline Fall & $\begin{array}{l}\text { Meßergebnis } \\
\text { einer validen } \\
\text { Messung }\end{array}$ & $\begin{array}{l}\text { Varianzanteil } \\
\text { (Kessler- } \\
\text { Greenberg) } \\
\text { ind }\end{array}$ & $\begin{array}{l}\text { normierter } \\
\text { Varianzanteil } \\
\text { (Kirschner) } \\
\text { rel }\end{array}$ & $\begin{array}{l}\text { Individual- } \\
\text { index }\end{array}$ & $\mathrm{IK}\left(\mathrm{x}_{1}, \mathrm{x}_{2}\right)$ & $\mathrm{b}\left(\mathrm{x}_{2}, \mathrm{x}_{1}\right)$ & $\mathrm{s}\left(\mathrm{x}_{2}\right) / \mathrm{s}\left(\mathrm{x}_{1}\right)$ \\
\hline Schaubild 1a & $\times$ & $\times$ & $\times$ & $\times$ & 1 & 1 & 1 \\
Schaubild 1b & 100 & 50 & 0 & 100 & 0 & 0 & 1 \\
Schaubild 1c & 100 & 75 & 50,5 & 100 & 0,5 & 0,5 & 1 \\
Schaubild 1d & 0 & 0 & 0 & 0 & 1 & 2 & 2 \\
Schaubild 2a & $0<2 \mathrm{a}<100$ & 100 & 100 & 42,6 & 0,5 & 1 & 2 \\
Schaubild 2b & $2 \mathrm{~b}=2 \mathrm{a}$ & 25 & 100 & 42 & 0,5 & 0,25 & 0,5 \\
Schaubild 2c & $2 \mathrm{c}<2 \mathrm{a}$ & 96,4 & 96,3 & 33,4 & 0,5 & 1,5 & 3 \\
Schaubild 2d & $2 \mathrm{~d}>2 \mathrm{a}$ & 80,0 & 0 & 70,9 & 0 & 0 & 2 \\
\hline
\end{tabular}

Quelle: eigene Berechnungen

ind noch rel geben jedoch diesen Fall richtig wieder - der Wert von ind ist sogar mit 50 Prozent immer noch ,zutreffender" als der von rel mit 0 Prozent (s. a. Tabelle 1). Schaubild 1c stellt eine Abwandlung von Schaubild $1 \mathrm{~b}$ mit nur noch zwei Fällen von Veränderung dar - die (geringere) Veränderung ist nach wie vor rein individueller Natur. Trotzdem weisen ind und rel mit 75 bzw. 50,5 Prozent vollkommen unterschätzte IndividualKomponenten aus. In Schaubild 1d ist die Veränderung vollkommen struktureller Natur - hier wird die Individualkomponente jeweils zutreffenderweise mit 0 wiedergegeben. ${ }^{4}$ Sowohl die KesslerGreenberg-Zerlegung wie aber auch das normierte $\mathrm{Ma} ß$ von Kirschner geben somit gerade die Hälfte der bislang besprochenen Extremfälle richtig wieder.

In den Schaubildern 2a-d wird der Frage nachgegangen, ob markante Unterschiede von Veränderung wenigstens in einem ordinalen Sinne zutreffend gemessen werden. In Schaubild 2a ist - verglichen mit Schaubild $1 \mathrm{~d}$ - ein größerer Anteil individueller Veränderung zu erwarten. Der Anteil individueller Veränderung ist jedoch aufgrund des ..Herausdrehens" unter dem von Schaubild $1 \mathrm{c}$ anzunehmen. Er müßte also zwischen 0 und 100 liegen. Beide Maße weisen dennoch den unzutreffenden Wert von 100 Prozent aus (Tabelle 2). Schaubild $2 b$ unterscheidet sich von Schaubild $2 a$ nur durch die Richtung des Herausdrehens - es ist also derselbe Individualanteil zu erwarten. Nur rel

\footnotetext{
'bei Definition von rel und indmin aus dem Wertebereich $0 \leq \mathrm{r}\left(\mathrm{x}_{1}, \mathrm{x}_{2}\right) \leq 1$
}

behält aber den (falschen) Wert von 100 Prozent, während ind den Wert von 25 Prozent annimmt. Vergleicht man die Schaubilder $2 \mathrm{a}$ und $2 \mathrm{c}$, so nimmt nur die strukturelle Komponente zu, worauf richtigerweise ind und rel einen niedrigeren Wert annehmen. Beim Schaubild $2 \mathrm{~d}$ ist gegenüber Schaubild 2a mit einer höheren Individual-Komponente zu rechnen. Der Wert von ind könnte zwar mit 80 Prozent in einer annähernd zutreffenden Größenordnung liegen, in Schaubild 2 a hatte jedoch ind einen höheren Wert von 100 . Der Wert von rel liegt in Schaubild $2 \mathrm{~d}$ mit 0 gänzlich daneben. Auch markante Unterschiede werden demnach von beiden Maßzahlen nur teilweise richtig wiedergegeben.

Eine eingehendere Diskussion zeigt also, daß beide Maße schon im Extrembereich nur sehr unbefriedigend in der Lage sind, zwischen strukturellem und individuellem Wandel zu unterscheiden. Insbesondere genießt auch das von Kirschner entwickelte Maß im Rahmen der vorab zugrunde gelegten Extrem-Kriterien nur den einzigen Vorzug der Symmetrie: Symmetrisches Herausdrehen führt bei gleichem Korrelationskoeffizienten, wie in den Schaubildern $2 \mathrm{a}$ und $2 \mathrm{~b}$ illustriert, zu einem gleichen Wert von rel.

\subsection{Eine Validitätsbeurteilung anhand der generellen Eigenschaften}

Ein erneuter Blick in die Tabelle 1 gibt über einige generellere Eigenschaften von ind und rel Auskunft. Für jedes Verhältnis der Standardabweichungen ist der Maximalwert von ind und rel bei einem Korrelations-Koeffizienten größer als 0 und 
2a)
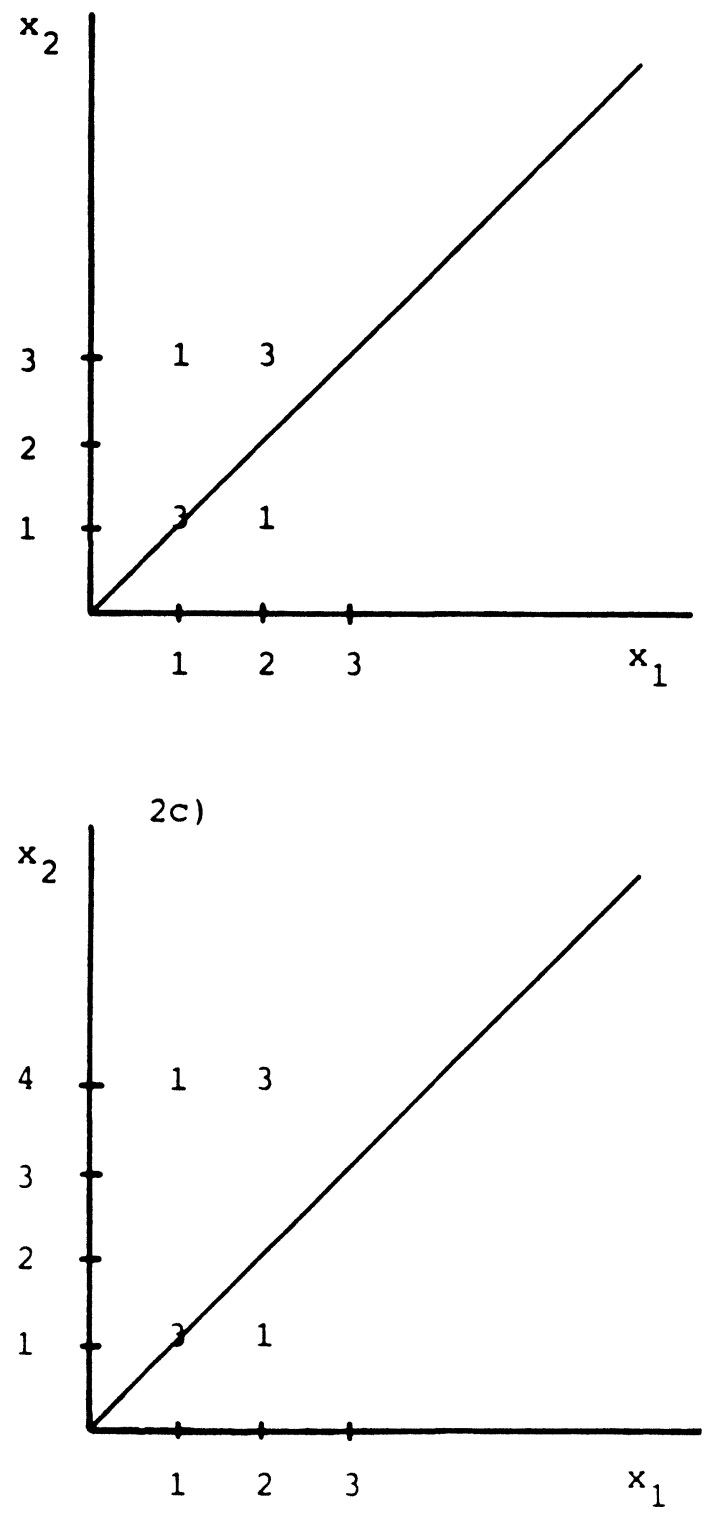

2b)

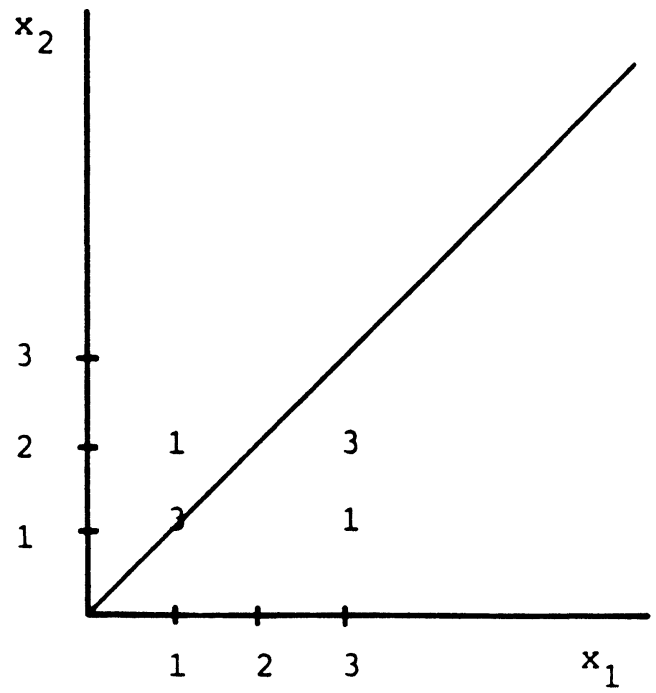

2d)

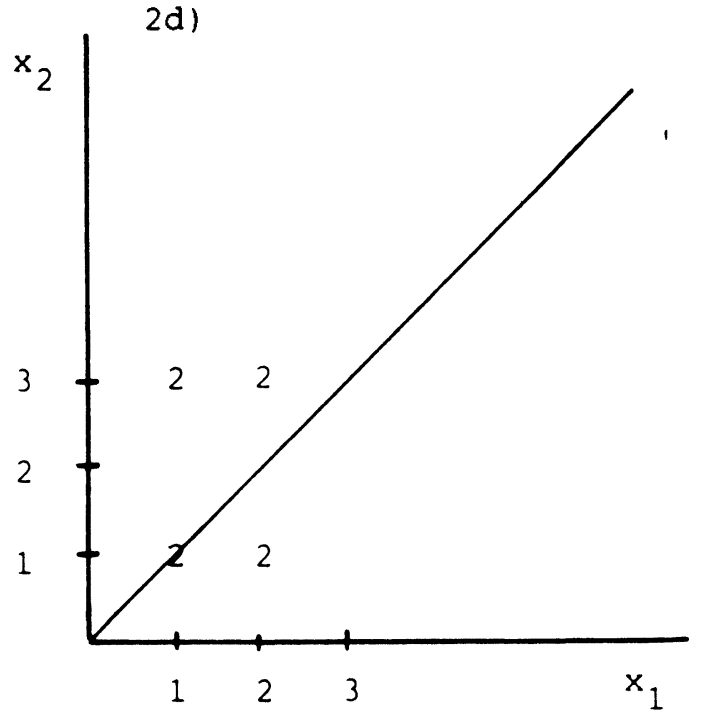

Schaubild 2 Extrembeispiele für Veränderungen zwischen zwei Meßzeitpunkten.

Jeweils sind 8 Wertepaare dargestellt. Die Zahlen geben die Häufigkeit der jeweiligen Wertekombination wieder, also: 1 = 1 Beobachtung, 2 = 2 Beobachtungen usw.

Quelle: eigene Zusammenstellung 
kleiner als 1 angesiedelt ${ }^{5}$, da nämlich, wo der Korrelationskoeffizient den Wert bzw. den Kehrwert des Verhältnisses der Standardabweichungen annimmt. Für $s\left(x_{2}\right)>s\left(x_{1}\right)$ ist der Maximalwert jeweils 100. Bei $s\left(x_{2}\right)<s\left(x_{1}\right)$ ist er für ind kleiner als 100 und gleich dem Produkt aus dem Standardabweichungsverhältnis und dem Korrelationskoeffizienten, für rel dagegen wegen der Normierung nach wie vor 100 (Prozent des Wertebereichs von ind). Wenn man berücksichtigt, daß ein sinkender Korrelationskoeffizient bei festem Varianzverhältnis mit einer durchweg steigenden Individualkomponente einhergehen sollte, so stehen ind und rel auch $z$ wischen den zuvor betrachteten Extremfällen nur zum Teil in einer validen Größenordnung.

Betrachtet man für jedes Verhältnis der Standardabweichungen den Minimalwert von ind bei variabler Korrelation, so nimmt ind, wie aus Formel (2) und Tabelle 1 ersichtlich, dann den Wert 0 an, wenn $\mathbf{r}\left(\mathrm{x}_{1}, \mathrm{x}_{2}\right)$ den Wert 1 hat. Auch der Wert von rel nimmt vom Maximalwert an mit steigender Korrelation ab, wird jedoch von Kirschner (1986) nur bis zu einem Korrelationskoeffizienten von 0,95 berechnet, wobei auch indmin und indmax auf diesen Bereich bezogen sind. Wie schon bei der Besprechung von Schaubild 1 a erwähnt und aus Gleichung (3) leicht ersichtlich, ist jedoch der Wert von rel von dem Minimalwert und, weil dieser „am Rand“ auftritt, von dem berücksichtigten Wertebereich abhängig. Eine Erweiterung des Wertebereichs führt deshalb zu einer Erhöhung von rel wie aus der jeweils dritten Zeile von Tabelle 1 ersichtlich. Wird ein Wertebereich bis zum Korrelationskoeffizienten von 1 zugelassen, so ist rel für den Bereich $\mathrm{s}\left(\mathrm{x}_{2}\right) / \mathrm{s}\left(\mathrm{x}_{1}\right) \geq 1$ mit ind identisch, da sich auch der Wertebereich von ind bis 100 Prozent erstreckt. Eine theoretische Begründung für die Einengung des berücksichtigten Wertebereichs ist nicht ersichtlich. Auch die von Kirschner (1986: 28, 30) gelieferte Begründung, daß man bei hohen Korrelationskoeffizienten ohnehin nicht von untersuchenswerten Veränderungen reden könne, ist nicht zugkräftig - schließlich kann sich ein beträchtliches Ausmaß von Veränderung bei einem Korrelationskoeffizienten von 1 in Form einer Geraden manifestieren, die wesentlich steiler oder flacher als die Winkelhalbierende verläuft.

\footnotetext{
Negative Korrelationskoeffizienten werden auch von Kirschner (1986) nicht in Betracht gezogen und sind forschungspraktisch von geringer Relevanz für den $\mathrm{Zu}$ sammenhang der Meßwerte ein und desselben Merkmals zu zwei Meßzeitpunkten.
}

Betrachten wir noch abschließend den Wertebereich von ind und rel bei variablem Verhältnis der Standardabweichungen und konstanter Korrelation. Die höchste Individualkomponente ist, wie im Fall eines Streifens um die Winkelhalbierende, bei einer im zweiten Zeitpunkt unveränderten Varianz anzunehmen. ${ }^{6}$ Weder ind noch rel haben jedoch ihren höchsten Wert bei einem Verhältnis der Standardabweichungen von 1. Bei einer Abweichung von 1 ist ferner mit einer symmetrisch abnehmenden Individualkomponente zu rechnen nur das normierte Maß von Kirschner weist jedoch symmetrische Werte auf.

Auch die Betrachtung der generellen, nicht nur auf Extremfälle bezogenen Eigenschaften von ind und rel offenbart damit eine geringe Validität beider Maße bei der Trennung von individueller und struktureller Veränderung. Es besteht daher weiterhin Bedarf nach einer alternativen Maßzahl. Eine solche wird im folgenden entwickelt.

\section{Ein alternatives Maß für die Trennung struktureller und individueller Veränderung}

Angesichts der vorangegangenen Diskussion scheint ein alternatives Maß für die Trennung struktureller und individueller Veränderung angebracht. Als Grundlage für die Entwicklung einer neuen Maßzahl ist eine nochmalige Revision der Kessler-Greenberg-Zerlegung und des normierten Maßes von Kirschner und eine Beantwortung der Frage sinnvoll: Warum liefern beide Maße derart offensichtlich unzutreffende Ergebnisse?

Eine Antwort auf diese Frage ist aus Tabelle 3 ersichtlich. In Tabelle 3 sind, wie in Tabelle 1, die Werte für ind und rel bei unterschiedlicher Korrelation ausgewiesen, nunmehr aber nicht nach dem Verhältnis der Standardabweichungen, sondern nach dem Regressionskoeffizienten aufgeschlüsselt. Es fällt sofort ins Auge, daß sich Maximalwerte für ind und rel jeweils bei einem Regressionskoeffizienten von 1 errechnen, während eine Abweichung von 1, egal in welche Richtung, mit einer niedrigeren Individualkomponente einhergeht. Das „Herausdrehen“ wird demnach von beiden Maßen an den Regressionsgeraden gemessen.

Es ist jedoch leicht einzusehen, daß die Regressionsgerade einen ungeeigneten Anhaltspunkt für

${ }^{6}$ unter Ausschluß rein additiver Struktureffekte 
Tabelle 3 Der Kessler-Greenberg-Anteil individueller Varianz (ind), das normierte Maß für individuelle Veränderung (rel) und der Individualindex für die Komponente individueller Veränderung (IK) nach dem Korrelationskoeffizienten und dem Regressionskoeffizienten.

Korrelations- Regressionskoeffizient $b\left(x_{2}, x_{1}\right)=$ koeffizient

\begin{tabular}{|c|c|c|c|c|c|c|c|c|c|c|c|c|c|}
\hline$r\left(x_{1}\right.$, & $\left.x_{2}\right)$ & 0.00 & 0.20 & 0.40 & 0.60 & 0.80 & 1.00 & 1.20 & 1.40 & 1.60 & 1.70 & 1.80 & 2.00 \\
\hline 0.0 & IND & $50.0^{11}$ & $x$ & $x$ & $x$ & $x$ & $x$ & $x$ & $x$ & $x$ & $x$ & $x$ & $x$ \\
\hline & REL .95 & $x$ & $x$ & $x$ & $x$ & $x$ & $x$ & $x$ & $x$ & $x$ & $x$ & $x$ & $x$ \\
\hline & REL 1.0 & $x$ & $x$ & $x$ & $x$ & $x$ & $x$ & $x$ & $x$ & $x$ & $x$ & $x$ & $x$ \\
\hline & IK & $100.0^{1)}$ & $x$ & $x$ & $x$ & $x$ & $x$ & $x$ & $x$ & $x$ & $x$ & $x$ & $x$ \\
\hline 0.2 & IND & $x$ & 60.0 & 91.4 & 98.2 & 99.7 & 100.0 & 99.9 & 99.7 & 99.4 & 99.3 & 99.2 & 99.0 \\
\hline & REL .95 & $x$ & 20.2 & 90.7 & 98.1 & 99.7 & & .9 & 7 & & & & 8.9 \\
\hline & REL 1.0 & $x$ & 60.3 & 91.4 & 98.2 & 99.7 & 100.0 & 99.9 & 99.7 & 4 & 3 & 2 & 99.0 \\
\hline & IK & $x$ & 100.0 & 66.1 & 57.5 & 53.8 & 51.7 & 50.3 & 49.4 & 48.7 & 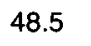 & 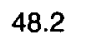 & 47.8 \\
\hline 0.4 & IND & $x$ & 24.7 & 70.0 & 92. & 98.8 & 1 & 995 & 98. & 9 & 9 & 4 & 95.5 \\
\hline & REL. & $x$ & & 40 & 9 & 9 & 1 & & & & & & 5.3 \\
\hline & REL 1.0 & $x$ & 98.8 & 70.4 & 92. & 98.8 & 100.0 & & 9 & 9 & & & 5.5 \\
\hline & IK & $x$ & 59.4 & 100.0 & 70.5 & 59.4 & 53.8 & 50.4 & 48. & 46. & 0 & 4 & 4.5 \\
\hline 0.5 & IND & $x$ & 15.8 & 57.1 & 87.1 & 98.0 & 100.0 & 99.1 & 97.4 & 95.5 & 94.7 & 93.8 & 92.3 \\
\hline & REL. .95 & $x$ & 98.6 & 834 & 76.6 & 97.7 & 10 & 99.0 & 97.2 & 95 & 94.4 & 93 & 92.0 \\
\hline & REL 1.0 & $x$ & 98.7 & 89.3 & 87.1 & 98.0 & 100.0 & 99.1 & 97.4 & 95.5 & 94.7 & 93.8 & 92.3 \\
\hline & IK & $x$ & 49.2 & 78.0 & 81.2 & 63.4 & 55.0 & 50.1 & 47.0 & 44.9 & 44.0 & 43.3 & 42.1 \\
\hline 0.6 & IND & $x$ & 10.0 & 44.1 & 80.0 & 96.6 & 100.0 & 98.5 & 95.6 & 92.7 & 91.3 & 90.0 & 87.7 \\
\hline & REL .95 & $x$ & 89.5 & 99.2 & 60.6 & 95.4 & 100 & 98 & 95.3 & 92.3 & 90.9 & 89.5 & 87.2 \\
\hline & REL 1.0 & $x$ & 90.0 & 99.3 & 80.4 & 96.6 & 100.0 & 98.5 & 95.6 & 92.7 & 91.3 & 90.0 & 87.7 \\
\hline & IK & $x$ & 40.4 & 61.4 & 100.0 & 68.9 & 56.2 & 49.4 & 45.2 & 42.4 & 41.3 & 40.4 & 38.9 \\
\hline 0.8 & INL & $x$ & 3.4 & 20.0 & 50 & 90.0 & 100.0 & $5 ?$ & 87.3 & 80. & , & 7 & 69.2 \\
\hline & REL .95 & $x$ & 52.7 & 78.3 & 99.1 & 80.8 & 100.0 & 94.4 & 85.9 & 78.3 & 75.1 & 72.3 & 67.5 \\
\hline & REL 1.0 & $x$ & 54.3 & 80.0 & 99.3 & 90.5 & 100.0 & 95.3 & 87.3 & 80.0 & 76.8 & 74.0 & 69.2 \\
\hline & IK & $x$ & 22.5 & 32.8 & 52.5 & 100.0 & 58.7 & 44.3 & 37.1 & 32.8 & 31.3 & 30.0 & 28.0 \\
\hline 1.0 & IND & $x$ & 0. & 0 & 0 & 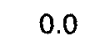 & $x$ & 0. & 0.0 & 0.0 & 0.0 & 0.0 & 0.0 \\
\hline & REL. .95 & $x$ & 0.0 & 0.0 & 0.0 & 0. & $x$ & 0. & 0.0 & 0.0 & 0.0 & 0.0 & 0.0 \\
\hline & REL 1.0 & $x$ & 0.0 & 0.0 & 0.0 & 0.0 & $x$ & 0.0 & 0.0 & 0.0 & 0.0 & 0.0 & 0.0 \\
\hline & IK & $x$ & 0.0 & 0.0 & 0.0 & 0.0 & $x$ & 0.0 & 0.0 & 0.0 & 0.0 & 0.0 & 0.0 \\
\hline
\end{tabular}

1) bei einem Varianzverhältnis von 1

Quelle: eigene Berechnungen

die Trennung von individueller und struktureller Veränderung darstellt. Betrachtet man beispielsweise Schaubild $1 \mathrm{~b}$, dort ist der Regressionskoeffizient 0 (siehe auch Tabelle 2), so müßte man anhand des Regressionskoeffizienten auf ein starkes „Herausdrehen“ schließen, obwohl ganz offensichtlich keinerlei systematische Veränderung vorliegt. Dies ist auch algebraisch nachvollziehbar, wenn man sich vergegenwärtigt, daß sich der Regressionskoeffizient $b\left(x_{2}, x_{1}\right)$ gemä $\beta$

$$
b\left(x_{2}, x_{1}\right)=r\left(x_{1}, x_{2}\right) \cdot \frac{s\left(x_{2}\right)}{s\left(x_{1}\right)}
$$

aus den Standardabweichungen und der Korrelation berechnet. Der Regressionskoeffizient wird in Schaubild $1 \mathrm{~b}$ schon einfach deshalb 0 , weil keinerlei Systematik da ist, was sich in einem Korrelationskoeffizienten von 0 ausdrückt. Ein Regressionskoeffizient nahe 0 wird auf der anderen Seite nur dann ein echtes „Herausdrehen“ anzeigen. wenn alle Wertepaare mehr oder weniger auf der Regressionsgeraden liegen, $r\left(x_{1}, x_{2}\right)$ also nahe bei 1 liegt und $b\left(x_{2}, x_{1}\right)$ nur deshalb nahe bei 0 liegt, weil $s\left(x_{2}\right)$ einen Wert nahe 0 hat. Ausschlaggebend für die Trennung von strukturellem und individuellem Wandel ist also das Verhältnis der Standardabwei- 
chungen und nicht der von ind und rel berücksichtigte Regressionskoeffizient.

Auf der Grundlage dieser Erkenntnis wird hier zur Messung der Individualkomponente der folgende Index IK vorgeschlagen:

(5)

$\mathrm{IK}=\left(\frac{1-\mathrm{r}\left(\mathrm{x}_{1}, \mathrm{x}_{2}\right)}{\left(1-\mathrm{r}\left(\mathrm{x}_{1}, \mathrm{x}_{2}\right)\right)+\left(\frac{\left(\tan ^{-1}\left(\mathrm{~s}\left(\mathrm{x}_{2}\right) / \mathrm{s}\left(\mathrm{x}_{1}\right)-45\right)\right.}{45}\right)}\right)^{2}$

Zur besseren Vergleichbarkeit nimmt der Index wiederum auf die Individualkomponente Bezug; eine Strukturkomponente SK ist als Ergänzung zu 1 bzw., in Prozent ausgedrückt, zu 100, leicht berechenbar. Wir wollen fortan die Eigenschaften dieses Individualindex diskutieren. Der Individualindex ist, ebenso wie das Maß von Kirschner, nicht an eine mathematische Herleitung, vergleichbar der Kessler-Greenberg-Zerlegung, geknüpft. Er soll daher nur als Rangindex interpretiert werden. Er erweist sich aber dennoch als weitaus brauchbarerer Index für die Zerlegung von strukturellem und individuellem Wandel.

Gleichung (5) basiert auf dem Korrelationskoeffizienten als Maß für individuelle Veränderung und auf dem Verhältnis der Standardabweichungen als ein $\mathrm{Ma}$ für strukturellen Wandel, das - wie beschrieben - geeigneter ist als der Regressionskoeffizient. Das Verhältnis der Standardabweichungen wird über den Arcustangens in den zugehörigen Steigungswinkel übersetzt. Da letztlich für das „Herausdrehen“ der Winkel mit der Winkelhalbierenden relevant ist, ist es, wie in Gleichung (5) beschrieben, sinnvoll, nur auf die Differenz zu 45 Grad Bezug zu nehmen. Um ein entsprechendes „Herausdrehen“ nach oben und unten gleich zu behandeln, berücksichtigt der Individualindex nur den Betrag der Differenz, der auf einen Wertebereich zwischen 0 und 1 standardisiert ist. Auch der gesamte Individualindex bewegt sich zwischen 0 und 1 bzw. 0 und 100 Prozent: Bei einer Korrelation von 1 nimmt der Zähler den Wert 0 an, so daß die gesamte Veränderung zu 100 Prozent als struktureller Wandel ausgewiesen wird. Liegen alle Wertepaare auf der 45-Grad-Linie, so nimmt auch der zweite Summand im Nenner den Wert von 0 an, womit eine Individualkomponente des nicht stattfindenden Wandels sinnvollerweise nicht definiert ist. Bei allen Korrelationskoeffizienten gröBer gleich 0 und kleiner als 1 wird der Nenner um so größer und der Individualindex damit um so kleiner, je weiter die Punktwolke aus der Winkel- halbierenden herausdreht. Um in dem forschungsrelevanten Bereich hoher Korrelation und ähnlicher Standardabweichungen eine größere Differenzierung zu erhalten, ist eine Quadrierung des insoweit berechneten Wertes von IK sinnvoll.

Die aus Gleichung (5) erkennbaren Eigenschaften sind auch in Tabelle 1, jeweils in der vierten Zeile, wiedergegeben. In Anlehnung an die Diskussion der Kessler-Greenberg-Zerlegung und des normierten Maßes von Kirschner ist sofort einsichtig. da $\beta$ der Index für die Individualkomponente der Veränderung zwischen zwei Meßzeitpunkten einer Panelbefragung viele erwünschte Eigenschaften aufweist. Auch in der Konfrontation mit den beschriebenen Extremfällen erweist sich der Index durchweg geeignet; siehe Tabelle 2.

Trotzdem sind abschließend einige Hinweise für die zutreffende Interpretation des vorgeschlagenen Individualindex angebracht. Zunächst ist nochmals darauf hinzuweisen, daß durch den Individualindex bzw. den korrespondierenden Strukturindex wie auch durch ind und durch rel - nicht das Ausma $ß$ an individueller bzw. struktureller Veränderung, sondern die Individual- bzw. Strukturkomponente der (gleich in welchem Ausmaß) stattfindenden Veränderung indiziert werden soll. Die Individualkomponente sollte dabei nicht als Anteil, sondern lediglich als ordinales $\mathrm{MaB}$ interpretiert werden. Der Individualindex informiert also darüber, ob und in welchen durch eine empirische Erfassung abgedeckten Bereichen sozial-struktureller Wandel festgestellt werden kann und im Hinblick auf seine Ursachen untersucht werden sollte, solange nicht das gesamte Ausmaß von Veränderung vernachlässigbar gering ist.

\section{Schlußfolgerungen}

Wie durch den Kessler-Greenberg-Anteil individueller Veränderung und das normierte $\mathrm{Maß}$ von Kirschner wird auch durch den Individual- bzw. Strukturindex auf die Deutlichkeit hingewiesen, mit der sozialer Wandel aus den auch individuellen Veränderungen hervortritt. Die angesprochene Deutlichkeit ist weniger für die Zurechenbarkeit des strukturellen Wandels zu bestimmten Ursachen als vielmehr per se von Bedeutung. ${ }^{7}$ In vielen

\footnotetext{
${ }^{7}$ Individueller Wandel ist nicht notwendigerweise mit zufälligem Wandel identisch; rein individueller Wandel besagt lediglich, daß per saldo keine sozialstrukturelle Veränderung stattgefunden hat.
} 
sozialstrukturellen Untersuchungen ist nicht nur (und häufig nicht einmal hauptsächlich) das Ausmaß der Veränderung von Interesse, das per saldo zwischen zwei Zeitpunkten stattgefunden hat. Von grundlegendem Interesse ist vielmehr häufig die Frage nach dem Ausmaß der gegenläufigen, individuellen Veränderungen, die gleichzeitig stattgefunden haben. Eine hohe Individualkomponente besagt beispielsweise, daß die Wahl der Erhebungszeitpunkte besonders wichtig und das per saldo festgestellte Ausmaß an sozialstruktureller Veränderung besonders wenig gesichert ist, wenn die individuellen Veränderungen nicht gleichmäBig über den Untersuchungszeitraum verteilt sind. Ein hoher Wert des Individualindex kann aber darüber hinaus auch zu recht unterschiedlichen sozialpolitischen Schlußfolgerungen führen. Findet beispielsweise zwischen zwei Meßzeitpunkten eine Verminderung von Armut um 100000 Personen statt, so können die Schlußfolgerungen unterschiedlich aussehen, je nachdem, ob nur diese 100000 Personen (durch eine sozialpolitische Maßnahme oder was immer) der Armut entkommen sind, oder ob gar 500000 eine Einkommensverbesserung erfahren haben, die sie über den Armutsbereich hinaushebt, wobei jedoch in demselben Zeitraum 400000 Personen verarmt sind.

Es sei schließlich auch darauf hingewiesen, daß der Individualindex auf absolute Veränderungen hin konzipiert ist. Veränderungen der Einkommen beispielsweise werden zu 100 Prozent als individuelle Veränderung verbucht, wenn sich die Aufund Abwärtsbewegungen ausgleichen und die Einkommensverteilung unverändert bleibt, während sozialstruktureller Wandel nur ausgewiesen wird, wenn sich die Einkommensverteilung ändert. ${ }^{8}$

Abschließend sei erwähnt, daß der Individualindex nur unter Vorbehalt als Index für mangelnde (Retest-)Reliabilität des Erhebungsinstruments gewertet werden darf. Er weist lediglich darauf hin, in welchem Maße die Veränderungen gegenläufig sind. Individuelle Veränderungen sind nicht notwendigerweise als zufällige Veränderungen unreliabler Frage zu interpretieren; vielmehr können gegenläufige Tendenzen bei den verursachenden Größen vorliegen.

${ }^{8}$ Dies bedeutet natürlich, daß sich die Wahrscheinlichkeit des Übergangs von einer Einkommensklasse in eine andere umgekehrt proportional zu der Besetzung der Klasse verhält.

\section{Literatur}

Kessler, R. C./Greenberg, D. F. 1981: Linear Panel Analysis. New York: Academic Press

Kirschner, H.-P. 1986: Zur Kessler-Greenberg-Zerlegung der Varianz der Meßdifferenz zwischen zwei Meßzeitpunkten einer Panel-Befragung. in: ZUMANachrichten Nr. 18/1986, 21-37

Tölke, A. 1980: Zuverlässigkeit retrospektiver Verlaufsdaten: Qualitative Ergebnisse einer Nachbefragung. Arbeitspapier Nr. 30 des Sonderforschungsbereich 3 